\title{
IMPACTO DE LA PREPARACIÓN DEL SUELO SOBRE LAS CARACTERISTICAS FISICAS DEL SUELO, CALIDAD Y RENDIMIENTO DE LAS RAICES DE YUCA (Manihot esculenta Crantz), VARIEDAD ALGODÓN, NUEVA GUINEA - RAAS
}

\section{IMPACT OF TILLAGE INTENSITY ON SOIL PHYSICAL CHARACTERISTICS, AND QUALITY AND YIELD OF CASSAVA ROOTS (Manihot esculenta Crantz), ALGODÓN VARIETY, NUEVA GUINEA - RAAS}

\section{Duarte-Centeno Nubia', Figueroa-Martínez Manuel' ${ }^{2}$, Hernández Luis ${ }^{3}$ Aguilar-Bustamante Víctor ${ }^{4}$} ${ }^{1}$ Ing. Agrónomo UNA Juigalpa, ${ }^{2}$ Ing. Agrónomo UNA Juigalpa, ${ }^{3}$ Ing. Docente UNA Managua, ${ }^{4} \mathrm{PhD}$. Docente UNA Managua

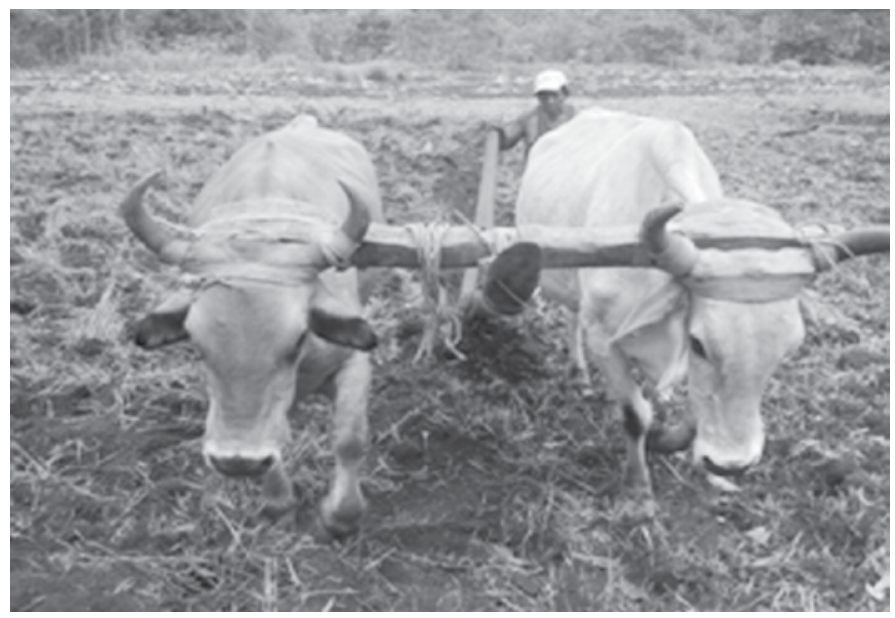

\section{RESUMEN}

El estudio se realizó en el Centro de Desarrollo Tecnológico (CDT) del Instituto Nicaragüense de Tecnología Agropecuaria (INTA) en Nueva Guinea, RAAS desde Octubre de 2005 a Agosto de 2006. El objetivo general fue evaluar cuatro sistemas de labranza sobre las propiedades físicas del suelo, el crecimiento y rendimiento del cultivo de la yuca variedad Algodón, así como la calidad de las raíces. Los tratamientos estudiados fueron: subsoleo más encamado (SE), grada más encamado (GE), cero labranza (CL) y tracción animal (TA). El diseño utilizado fue un arreglo de bloques completos al azar (BCA) con tres repeticiones. Las variables de suelo evaluadas fueron: densidad aparente, densidad real, porosidad total y capacidad de campo. Las variables agronómicas estudiadas fueron altura de planta, diámetro del tallo, peso fresco de la planta, número de raíces totales por planta, número de raíces exportables, longitud de raíces exportables, diámetro de raíces exportables, peso de raíces exportables, rendimiento de raíces exportables, rendimiento de raíces no exportables y rendimiento total de raíces. Para el análisis de los datos se usó el paquete estadístico SAS Institute V 8 a base de Análisis

\section{ABSTRACT}

The study was carried out in the National Institute of Agrarian Technology (INTA) Nueva Guinea from October 2005 to August 2006. The main objective was to evaluate the effect of four different soil preparation systems on soil physical properties, the growth, yield and quality of cassava root variety Algodón. Four soil tillage systems were studied: row subsoil plus ridge tillage (SE), terracing plus ridge tillage (GE), no tillage (CL), and animal traction (TA). The experiment was set up in a randomized complete block design (BCA) with three replicates. The soil variables measured were: bulk density, soil density, soil porosity and water field soil capacity, and the plant growth and yield variables were: plant height, stem diameter, plant fresh weight, total roots per plant, number of exportable roots, length of exportable roots, diameter of exportable roots, weight of exportable roots, yield of exportable roots per hectare, yield of non exportable root per hectare and total root yield per hectare. The analysis of variance (ANVA) was made using the Statistic Analysis Systems software (SAS Institute V8) multiple mean comparisons for TUKEY with $5 \%$ of error. In the Animal 
de Varianza (ANDEVA) y comparación de rangos múltiples de medias por TUKEY al 5\% de margen de error. El suelo preparado con tracción animal (TA), presentó los menores valores de densidad aparente y mayor porcentaje de porosidad del suelo. Las plantas de yuca alcanzaron la mayor altura, el mayor diámetro y mayor peso fresco con cero labranza (CL) y tracción animal (TA). El mayor número de raíces totales por planta fue alcanzado con tracción animal (TA) seguido de cero labranza $(\mathrm{CL})$. El mayor número de raíces exportables, el mayor diámetro de raíces exportables y peso de las raíces exportables fue alcanzado con el método cero labranza (CL) seguido de tracción animal (TA). El mayor rendimiento de raíces totales fue alcanzado en el suelo con Cero labranza (CL), así como el mayor rendimiento de raíces exportables y no exportables con una relación de $76 \%$ de raíces exportables. Palabras Clave: Labranza, intensidad de labranza, densidad aparente, porosidad del suelo.
Traction treatment (TA) were registered the lowest soil bulk density, the highest percentage of soil porosity distribution. The cassava plan reached the highest plant height, the highest stem diameter and the highest plant fresh weight in no tillage system (CL) followed by Animal traction (TA). The highest number of total cassava roots per plant was found in Animal traction system (TA) followed by no tillage system (LC). The highest number of exporter roots, the highest diameter of exporter roots and the weight of exporter roots were found in no tillage system (CL) followed by animal traction (TA). The highest total yield was reached by no tillage system (CL), also the yield of exporter roots and the yield of non exporter roots with a relationship of $76 \%$ of exportable roots. Keywords: Tillage, yield and quality, tillage intensity, bulk density, soil porosity.

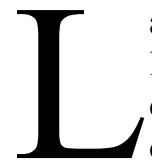

a yuca (Manihot esculenta Crantz) pertenece a la familia Euphorbiaceae, compuesta por más de 7,200 especies distribuidas en zonas cálidas de todo el mundo. Las raíces tuberosas de yuca son ricas en hidratos de carbono $(26.7 \%)$, buena fuente de vitamina B $(0.3 \mathrm{mg})$ y vitamina C (48.2 $\mathrm{mg})$, aunque sus raíces tienen un bajo contenido de proteínas $(3.1 \%)$ y en grasa $(0.4 \%)$ sus hojas presentan un $17 \%$ de proteína (Ospina y Ceballos 2002). La yuca es un producto agrícola de vital importancia para la seguridad alimentaria de muchos países del mundo. Es considerado el cuarto producto básico más importante después del arroz, el trigo y el maíz y su importancia también radica porque es fuente económica de calorías, especialmente para las personas de pocos recursos económicos y es el componente básico de la dieta de más de 1000 millones de personas en el mundo (FAO, 2000).

En Nicaragua el $70 \%$ de la producción de yuca se concentra principalmente en Nueva Guinea y el 30\% restante en los departamentos de Masaya y León donde se cultivan cada año alrededor de 17142 hectáreas a nivel nacional (INEC, 2001). Los rendimientos obtenidos por los productores nicaragüenses son de 6.82 ton/ha, considerado como muy bajo, comparado con su rendimiento potencial que es de 30 ton/ha.

Como una prueba más de las tecnologías existentes y probadas que arrojan como resultado aumento de la calidad y la producción y por ende ganancias económicas conservando los recursos naturales como es el recurso suelo, se estableció este ensayo en la finca demostrativa de Auxilio Mundial del municipio de Nueva Guinea financiado por la Empresa Desarrollo Agrícola Sociedad Anónima, acopiadora de Raíces y tubérculos (DAISA) de Nueva Guinea.

\section{MATERIALES Y MÉTODOS}

Descripción del lugar. El presente trabajo se realizó en la finca demostrativa de Auxilio Mundial, municipio de Nueva Guinea, RAAS. La precipitación acumulada de octubre a diciembre 2005, fue de $536 \mathrm{~mm}$ y de enero a agosto de 2006 fue de $1531 \mathrm{~mm}$. Las precipitaciones de la zona en estudio promedian anualmente $3270 \mathrm{~mm}$ con un mínimo de $3000 \mathrm{~mm}$ y un máximo de $3540 \mathrm{~mm}$. La temperatura promedio durante el estudio fue de $25^{\circ} \mathrm{C}$ (INETER, 2006).

Los suelos son clasificados como Orthoxic Tropudults del orden ultisoles. Tienen un drenaje interno natural imperfecto a bien drenados, de profundos a muy profundos, en relieve de plano a muy escarpado. La fertilidad natural tiene valores de baja a media, con un contenido variable de aluminio. Se han desarrollado de rocas básicas, intermedias y ácidas, de sedimentos aluviales, coluviales y fluviales. Textura franco arcilloso y arcilloso, arcilloso a muy arcilloso en el subsuelo.

El contenido de materia orgánica varía de alto a muy bajo, el pH varía de muy fuertemente ácido a medianamente ácido, el porcentaje de saturación de bases es de muy bajo a medio, la capacidad de intercambio catiónico es de bajo a medio, el porcentaje de aluminio intercambiable es de bajo a muy alto, el porcentaje de hierro libre es de alto a bajo y el porcentaje de fósforo asimilable es de bajo a medio (Quintana, et al., 1983).

Descripción del experimento. El diseño utilizado fue un arreglo en BCA y tres repeticiones para cada uno de los cuatro tratamientos: SE: Subsoleo a $40 \mathrm{~cm}$ y formación de los camellones; GE: Gradeo y formación de los camellones; CL: Ahoyado con azadón y siembra de la estaca y TA: dos pases de arado egipcio y surcado con bueyes. La parcela experimental fue de $240 \mathrm{~m} 2$ y la parcela útil de $38 \mathrm{~m} 2$. Cada uno de los 3 bloques fue de $960 \mathrm{~m} 2$ para un total de $2880 \mathrm{~m} 2$. 
Densidad aparente. Se realizó un muestreo cada $10 \mathrm{~cm}$ hasta los $50 \mathrm{~cm}$ de profundidad por calicata por tratamiento. Para el muestreo de campo se utilizó un cilindro de $100 \mathrm{~cm}^{3}$, y luego estas muestras fueron trasladadas al laboratorio de la UNA para sus respectivos análisis gravimétricos. Se introdujeron al horno durante 24 horas a una temperatura de $105{ }^{\circ} \mathrm{C}$. Se tomaron un total de 75 muestras con tres réplicas por profundidad y expresado en $\mathrm{g} / \mathrm{cm}^{3}$.

Para el cálculo de la densidad aparente se utilizó la siguiente fórmula (Sampat Gavande 1991):

$$
\begin{aligned}
& \mathrm{Da}=\mathrm{ODW}-\mathrm{RF}-\mathrm{CW} \\
& \mathrm{CV}-(\mathrm{RF} / \mathrm{PD})
\end{aligned}
$$

Densidad real. Se realizó con el método del picnómetro el cual se pesa vacio, después se pesan $2 \mathrm{~g}$ de suelo, al suelo se le agrega agua hasta taparlo y que le quede una película sobre nadante, después se pone a hervir, se bota el lodo en un balde (nunca en el desagüe del laboratorio), el picnómetro se lava, se afora con agua hervida y se pesa. Para este cálculo se utilizó la siguiente fórmula (Sampat Gavande 1991): $\mathrm{E}=(\mathrm{B}-\mathrm{A}) / \mathrm{A}+\mathrm{C})-\mathrm{B}+\mathrm{D})$

$$
\begin{aligned}
& \text { Porosidad total }(\%) . \% \mathrm{Pt}=(1-\mathrm{da} / \mathrm{dr}) * 100 \\
& \text { Donde: } \\
& \% \mathrm{Pt}=\text { porciento de porosidad total } \\
& \mathrm{da}=\text { densidad aparente } \\
& \mathrm{dr}=\text { densidad real }
\end{aligned}
$$

Capacidad de campo. Fue realizada utilizando el método de prensas Richard en laboratorio, aplicándose una presión de 1/3 de atmósfera. Las muestras fueron tomadas a las profundidades descritas anteriormente. Para el cálculo de la capacidad de campo se utilizo la siguiente fórmula (Sampat Gavande 1991):

$$
\begin{aligned}
\mathrm{H}_{2} \mathrm{O} \%= & (\mathrm{M}(\mathrm{S}+\mathrm{W})-\mathrm{M} \mathrm{S}) \quad \times 100 \\
& (\mathrm{MS}-\mathrm{MC})
\end{aligned}
$$

Donde:

$\mathrm{H}_{2} \mathrm{O} \%=$ Porcentaje gravimétrico de contenido de agua

$\mathrm{M}(\mathrm{S}+\mathrm{W})=$ Peso del suelo $+\mathrm{H}_{2} \mathrm{O}+$ lata

Ms = Peso del suelo + lata

$\mathrm{Mc}=$ Peso de la lata

Variables al cultivo. Altura de la planta $(\mathrm{cm})$, Diámetro del tallo $(\mathrm{mm})$, Peso fresco de las plantas $(\mathrm{kg} / \mathrm{ha})$, Número de raíces totales por planta, Número de raíces exportables por planta, Longitud de las raíces exportables ( $\mathrm{cm}$.), Diámetro de las raíces exportables $(\mathrm{cm})$, Peso de raíces exportables $(\mathrm{g})$, Rendimiento de las raíces exportables ( $\mathrm{kg} / \mathrm{ha})$, Rendimiento de las raíces no exportables y rendimiento total de las raíces $(\mathrm{kg} / \mathrm{ha})$. Para hacer estas mediciones se tomaron 10 plantas de la parcela útil de cada parcela experimental. Para la discusión de los resultados se realizó Análisis de Varianza (ANDEVA) con una distribución de Bloques Completos al Azar (BCA) y prueba de rangos múltiples de
TUKEY a un nivel de 5\% de margen de error. El programa utilizado fue, SAS Institute V-9, Cary North Carolina, 2007.

Manejo agronómico. La distancia de siembra fue de 1.20 $\mathrm{m}$ entre surcos y $0.80 \mathrm{~m}$ entre plantas para una densidad poblacional de 10,375 plantas por hectárea. Los esquejes fueron seleccionados de plantas de 11 a 12 meses de edad con $20 \mathrm{~cm}$ de largo de modo que cada esqueje tuviera entre 3 y 5 yemas. La siembra fue manual y la desinfección de las estacas se realizó con el fungicida oxicloruro de cobre aplicado con bomba de mochila directamente a la estaca.

A los primeros 30 días se fertilizó con la fórmula 18-46-00, a los 90 días se fertilizó con la formula 1515-15 y finalmente a los 120 días se utilizó la fórmula 00-00-60. En total se aplicaron $37.4 \mathrm{~kg}$ de $\mathrm{N}, 69.5 \mathrm{~kg}$ P y $85 \mathrm{~kg}$ de K. Para el control de las malezas se usó el herbicida- fosfónico (Glufosinate Ammonium).

\section{RESULTADOS Y DISCUSIÓN}

Densidad aparente (DA). La densidad aparente del suelo, es la relación entre la masa secada al horno de las partículas de suelo y el volumen total, incluyendo el espacio poroso que ocupan (Forsythe, 1980). SegúnlaclasificaciónhechaporCairo(1995), para los suelos del trópico, la densidad aparente se considera baja cuando es menor de $1.0 \mathrm{~g} / \mathrm{cm} 3 \mathrm{y}$ alta cuando es mayor de $1.60 \mathrm{~g} / \mathrm{cm} 3$. Según esta información, las cuatro formas de preparación del suelo no afectaron la densidad aparente del suelo ya que los valores están por debajo de lo establecido.

Los sistemas de intensidad de labranza afectaron significativamente la densidad aparente del suelo $(\operatorname{Pr}>\mathrm{F}=$ 0.0001). El valor más alto se encontró en gradeo más encamado (GE) con $0.95 \mathrm{~g} / \mathrm{cm} 3$. Los valores más bajos se presentaron con tracción animal (TA) y subsoleo más encamado (SE) con 0.87 y $0.89 \mathrm{~g} / \mathrm{cm} 3$. A los 10 y los $40 \mathrm{~cm}$ de profundidad se encontró la mayor DA con 0.95 y $0.97 \mathrm{~g} / \mathrm{cm} 3$. A los 20 y 30 $\mathrm{cm}$ de profundidad se encontraron valores significativamente bajos de 0.89 y $0.87 \mathrm{~g} / \mathrm{cm} 3(\operatorname{Pr}>\mathrm{F}=0.0001)$ ver figura 1 .

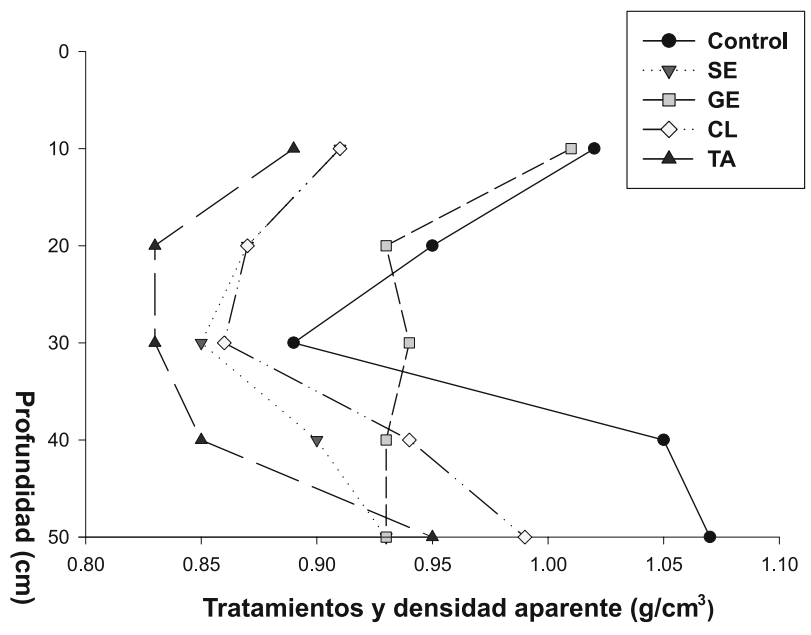

Figura 1. Efecto de sistemas de intensidad de labranza sobre la densidad aparente $\left(\mathrm{g} / \mathrm{cm}^{3}\right)$ del suelo, Nueva Guinea, 2006. 
Densidad real (DR). Constituye la fase sólida del suelo $\mathrm{y}$ es la relación entre la masa del suelo y la masa de igual volumen de agua. Al igual que la densidad aparente se expresa en $\mathrm{g} / \mathrm{cm}^{3}$. Los suelos con densidad real de $2.68 \mathrm{~g} /$ $\mathrm{cm}^{3}$ son suelos representativos de minerales como los silicatos y cuarzo, niveles normales de materia orgánica Cairo (1995). El mismo autor, también establece que valores menores a $2.40 \mathrm{~g} / \mathrm{cm}^{3}$ son bajos y valores mayores a 2.80 $\mathrm{g} / \mathrm{cm}^{3}$ son considerados altos. Los valores encontrados están por debajo de lo establecido por Cairo (1995). No hubo diferencia significativa entre tratamientos (figura 2)

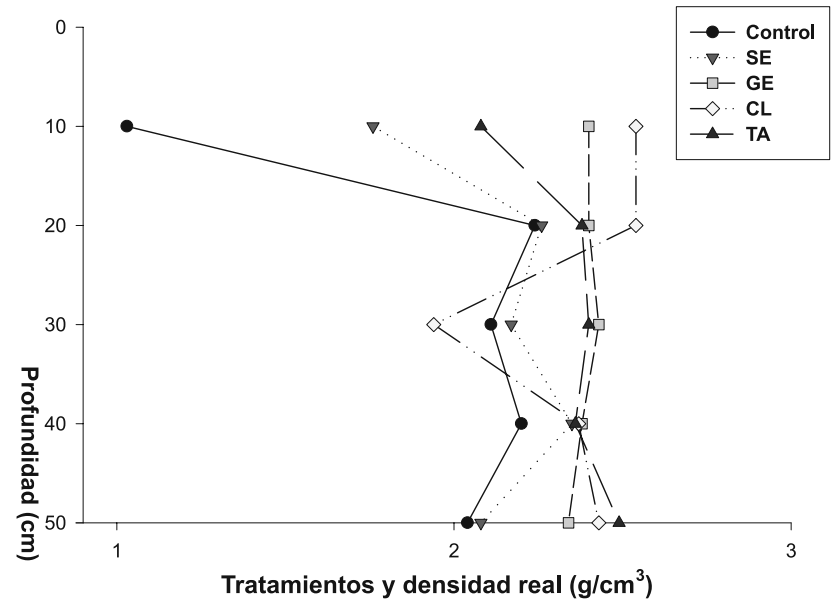

Figura 2. Efecto de los sistemas de intensidad de labranza sobre la densidad real del suelo, Nueva Guinea, 2006.

Porosidad total. Se define como estructura pobre a un arreglo de los agregados del suelo con un espacio poroso total menor o igual a $40 \%$. La porosidad está formada por la suma de los porcentajes de poros de diferentes tamaños. Los poros grandes sirven para aireación e infiltración, los poros medianos para la conducción de agua y los poros pequeños para el almacenamiento de agua disponible para la planta (Sampat y Gavande, 1991). En el presente trabajo se encontraron valores promedio de $59.4 \%$ de poros totales, lo que según Cairo (1995), los tratamientos no afectaron la porosidad del suelo.

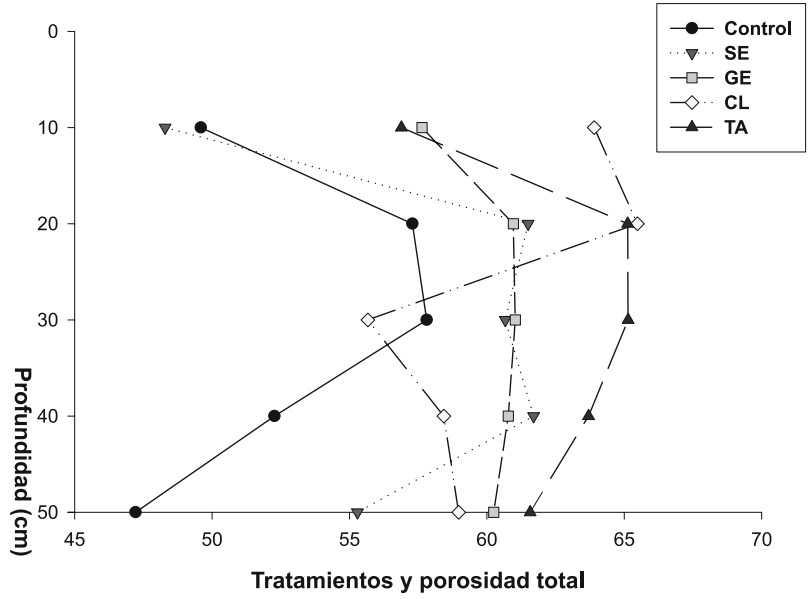

Figura 3. Efecto de los sistemas de labranza sobre la porosidad total del suelo, Nueva Guinea, 2006.
Se encontró diferencia significativa en el porcentaje de porosidad tanto entre los tratamientos como en la profundidad $(\operatorname{Pr}>\mathrm{F}=0.0001)$. Se presentó mayor porosidad en el tratamiento con tracción animal. La porosidad aumentó desde la superficie del suelo hasta los 30 $\mathrm{cm}$, pero disminuyó entre los 40 y $50 \mathrm{~cm}$ de profundidad.

Capacidad de campo. La capacidad de campo es la capacidad de agua que un suelo retiene contra la gravedad cuando se le deja drenar libremente. En un suelo bien drenado, por lo general se llega a este punto aproximadamente 48 horas después de irrigar. El promedio de los cuatro tratamientos fue de $43.48 \%$ considerada como buena ya que según (Cairo, 1995), los suelos con CC menores de $20 \%$ se considera baja y mayores de $55 \%$ es considerada como muy alta. El tratamiento, gradeo más encamado (GE) presentó menor capacidad de almacenamiento de agua y Subsoleo más Encamado (SE) mayor capacidad de almacenamiento de agua, seguido por tracción animal (TA) y Cero labranza (CL).

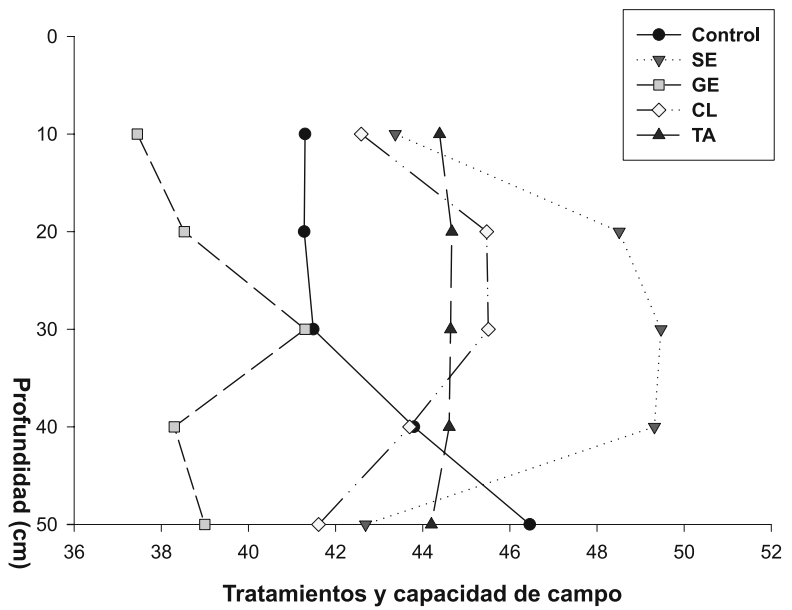

Figura 4. Efecto de sistemas de intensidad de labranza sobre la capacidad de campo del suelo, Nueva Guinea, 2006.

Altura de la planta. La yuca es un arbusto de tamaño variable de 1 a 5 metros de altura, agrupándose los cultivares en bajos cuando la altura es menor de 1.5 metros, intermedios entre 1.5 y 2.5 metros y altos cuando la altura es mayor de 2.5 metros (Montaldo, 1991). A los 90 días de establecido el experimento no se encontró diferencia estadística significativa en la altura entre tratamientos $(\mathrm{Pr}>\mathrm{F}=0.7052)$. Al finalizar el estudio ( 300 dds), el suelo con cero labranza, tracción animal y gradeo más encamado presentaron alturas similares, superiores $(\mathrm{Pr}>\mathrm{F}=$ 0.0237) a la altura de las plantas con Subsoleo más encamado.

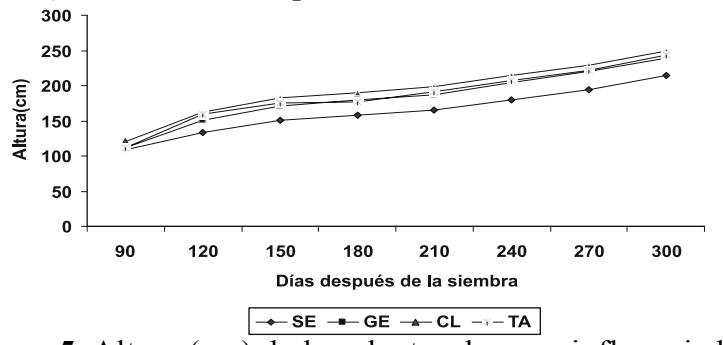

Figura 5. Altura $(\mathrm{cm})$ de las plantas de yuca influenciada por sistemas de intensidad de labranza, Nueva Guinea, RAAS. 
El suelo bajo labranza cero es menos compactado, posee mejor drenaje y poros más grandes lo que le permite almacenar agua para la época seca como febrero, marzo, abril y mayo en Nueva Guinea. Lo que significa que el suelo bajo Labranza cero amortiguó los efectos de este período seco expresándose en una mayor altura de las plantas de yuca.

Diámetro del tallo. El grosor del tallo de las plantas de yuca se asocia directamente con el rendimiento de las raíces. Un tallo con menos de $2 \mathrm{~cm}$ se puede considerar delgado, entre 2 y $4 \mathrm{~cm}$ se considera intermedio y mayor de $4 \mathrm{~cm}$ se considera grueso según (Montaldo, 1991). A lo largo del estudio no se encontró diferencias significativas en el diámetro de los tallos de las plantas de yuca. El suelo con Subsoleo más encamado presentó a lo largo del estudio los menores valores, seguido de gradeo más encamado.

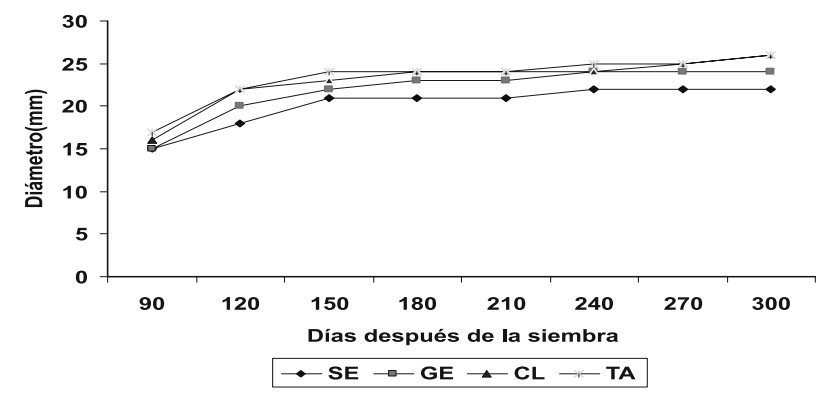

Figura 6. Diámetro (mm) de las plantas de yuca influenciado por sistemas de intensidad de labranza, Nueva Guinea, 2006.
El suelo bajo Cero Labranza y suelo preparado con Tracción Animal al final del estudio presentaron los mayores valores de diámetro del tallo. Se puede observar en la figura 6 , que los mayores valores de diámetro desde los 90 hasta los 270 días se presentaron en el suelo manejado con tracción animal.

Peso fresco de la planta. El conjunto de los tallos, ramificación y el IAF por parcela útil resulta el peso fresco de la planta de yuca. Según Montaldo (1991), se prefieren las variedades de ramificación alta para facilitar las labores agronómicas. El número de ramificaciones se reduce con bajos niveles de fertilidad del suelo y temperaturas bajas durante el ciclo del cultivo (CIAT, 1987). El tamaño de la hoja se reduce por falta de agua (Connor y Cock, 1981), bajas temperaturas (Irikura et al., 1979) y suelos con baja fertilidad (CIAT, 1987).

En la Tabla 1, se puede ver la diferencia significativa en el peso fresco de las plantas de yuca $(\operatorname{Pr}>\mathrm{F}=0.0059)$. El suelo con cero labranza y tracción animal, presentaron los mayores valores de peso fresco de las plantas, aunque estadísticamente igual al peso con grada + encamado. El suelo con subsoleo + encamado presentó las valores menores de peso freso por hectárea. Las variables número de raíces totales por planta, número de raíces exportables por planta, longitud de las raíces exportables, diámetro de las raíces exportables, y peso de raíces exportables no presentaron diferencia estadística significativa (Tabla 1).

Tabla 1. Variables de rendimiento del cultivo de la yuca influencias por sistemas de intensidad de labranza, Nueva Guinea, RAAS

\begin{tabular}{|c|c|c|c|c|c|c|}
\hline Tratamiento & $\begin{array}{l}\text { Peso fresco } \\
\text { de planta } \\
\left(\mathrm{kg} \mathrm{ha}^{-1}\right)\end{array}$ & $\begin{array}{l}\text { Raíces } \\
\text { totales por } \\
\text { planta }\end{array}$ & $\begin{array}{l}\text { Raíces } \\
\text { exportables } \\
\text { por planta }\end{array}$ & $\begin{array}{l}\text { Longitud } \\
\text { de raíz } \\
\text { exportable } \\
\text { (cm) }\end{array}$ & $\begin{array}{l}\text { Diámetro } \\
\text { de raíz } \\
\text { exportable } \\
(\mathrm{mm})\end{array}$ & $\begin{array}{l}\text { Peso de raíz } \\
\text { exportable } \\
\text { (g) }\end{array}$ \\
\hline Subsolado + encamado (SE) & 14546 & 4.8 & 2.8 & 34.5 & 5.84 & 631 \\
\hline Grada + encamado (GE) & 18046 & 5.8 & 3.2 & 33.1 & 5.91 & 650 \\
\hline Cero Labranza (CL) & 20990 & 5.9 & 3.8 & 33.8 & 6.14 & 732 \\
\hline Tracción animal (TA) & 22977 & 6.1 & 3.4 & 33.5 & 6.05 & 729 \\
\hline$\overline{\mathrm{Pr}}>\mathrm{F}$ & 0.0059 & 0.1204 & 0.1742 & 0.7595 & 0.6967 & 0.2749 \\
\hline
\end{tabular}

Nota: Cifras con la misma letra y sin letras son estadísticamente iguales.

Rendimiento de raíces exportables. Las raíces que poseen la longitud, diámetro y peso adecuados según las normas de exportación son separadas de las que no reúnen estos parámetros, luego son parafinadas y enviadas a los diferentes países como Puerto Rico y Estados Unidos. Las raíces parafinadas también son vendidas a nivel nacional a un precio por arriba de las no parafinadas. Aunque no se encontraron diferencias significativas entre tratamientos $(\operatorname{Pr}>\mathrm{F}=0.6094)$, el suelo manejado con cero labranza y tracción animal presentaron los mayores rendimientos de raíces exportables (Tabla 2). El suelo manejado con grada
+ encamado y subsolado + encamado presentaron los menores rendimientos de raíces exportables. Estos valores están por arriba de los encontrados por Chavarría (2003), en época de primera en Nueva Guinea con $17730 \mathrm{~kg} \mathrm{ha}^{-1}$.

Rendimiento de raíces no exportables. Las raíces que no poseen la longitud, diámetro y peso según las normas de exportación por la NCR, son destinadas al mercado local. La relación entre raíces exportables fue entre un 72 y un $77 \%$ del total de la producción y entre un 28 a un $23 \%$ de la raíces no exportables. El tratamiento que presentó mayor 
relación de raíces exportables fue el suelo con subsolado + encamado con $77 \%$ y tracción animal con $76 \%$. Los menores porcentajes fueron encontrados con grada + encamado con $72 \%$ y cero labranza con $73 \%$. En el rendimiento de raíces no exportables no se encontró diferencia estadística significativa $(\operatorname{Pr}>\mathrm{F}=0.6701)$. El suelo que presentó mayor rendimiento de raíces no exportables fue el manejado bajo cero labranza, seguido del suelo preparado con grada + encamado y tracción animal. El suelo con menor producción de raíces no exportables fue el subsolado + encamado (Tabla 2).

Rendimiento de raíces totales. El rendimiento de las raíces por planta es normalmente entre 3 y $5 \mathrm{~kg}$, pero existen algunos cultivares que aplicando una buena tecnología llega a producir hasta $10 \mathrm{~kg}$ (Montaldo, 1991). Se considera que un rendimiento fácil de obtener, con un paquete tecnológico adecuado es de 30 ton/ha de raíces a los 12 meses. Singh (1970), reporta rendimientos de 55 y 62 ton/ha en material de híbridos a los 11 meses de edad. Montaldo (1991) registra con la variedad UCV-2078, 68 ton/ha a los 16 meses y con UCV2194, 31 ton/ha a los 10 meses. Un estudio del CIAT (1982) indica que es posible obtener, con 22-28 oC de temperatura, precipitación de $1000 \mathrm{~mm}$ a los 12 meses de ciclo, con fertilidad alta del suelo, rendimientos de 35 ton/ha en promedio. Aburto (2004) en ensayos con 18 clones de yuca en el Centro Experimental de Occidente (CEO) Posoltega, donde incluyó la variedad Algodón, encontró un rendimiento de raíces exportables y no exportables (Tipo A y B) de 20,138.9 kg/ha, resultados que están por debajo de los encontrados en Nueva Guinea 2006. En los datos presentados en la tabla 2 , no se incluyen las raíces tuberosas muy pequeñas que son abandonadas en el campo (Moteras) pero representan una apreciable cantidad de entre un 3 a un 5\% del rendimiento total y que podrían ser utilizadas para preparar alimentos balanceados para los animales domésticos de familias pobres rurales. Los resultados de rendimiento total no presentaron diferencia estadística significativa. El tratamiento que presentó mayor rendimiento de raíces totales fue el suelo bajo cero labranza, seguido de tracción animal. Los menores valores fueron encontrados con grada + encamado y subsolado + encamado (Tabla 2).

Tabla 2. Variables de rendimiento del cultivo de la yuca influencias por sistemas de intensidad de labranza, Nueva Guinea, RAAS

\begin{tabular}{lccc}
\hline Tratamiento & $\begin{array}{c}\text { Rendimiento de raíces } \\
\text { exportables }\left(\mathrm{ka} \mathrm{ha}^{-1}\right)\end{array}$ & $\begin{array}{c}\text { Rendimiento de raíces } \\
\text { no exportables }\left(\mathrm{ka} \mathrm{ha}^{-1}\right)\end{array}$ & $\begin{array}{c}\text { Rendimiento de raíces } \\
\text { totales }\left(\mathrm{ka} \mathrm{ha}^{-1}\right)\end{array}$ \\
\hline Subsolado + encamado (SE) & 20773 & 6089 & 26862 \\
Grada + encamado (GE) & 19130 & 7334 & 26464 \\
Cero Labranza (CL) & 22529 & 8441 & 30970 \\
Tracción animal (TA) & 22086 & 7151 & 29238 \\
& & 0.6701 & 0.0689
\end{tabular}

\section{CONCLUSIONES}

El suelo preparado con tracción animal presentó los menores valores de densidad aparente lo que indica menor compactación del suelo, el suelo presentó una mayor cantidad y distribución del espacio poroso, así como una excelente capacidad de retención de agua dentro del perfil del suelo. El subsolado del suelo permitió una mayor retención de agua en el suelo.

Se observó una ligera compactación del suelo en los primeros $10 \mathrm{~cm}$ de profundidad y por debajo de los $40 \mathrm{~cm}$. En el espacio de 20 a $30 \mathrm{~cm}$ de profundidad donde mayormente se desarrollan las raíces no se encontró compactación ya que la densidad aparente fue alta.
El suelo bajo cero labranza y tracción animal, presentaron los valores más altos de altura de las plantas, mayor diámetro de los tallos de las plantas y el mayor peso fresco de plantas de yuca. El suelo preparado con tracción animal y labranza cero presentaron los valores más altos de número de raíces totales por planta, mayor número de raíces exportables por planta, mayor diámetro de raíces exportables por planta y mayor peso de las raíces exportables por planta.

El mayor rendimiento de raíces exportables y mayor rendimiento de raíces no exportable fue encontrado en el suelo preparado con cero labranza, seguido del suelo preparado con tracción animal. 


\section{REFERENCIAS BIBLIOGRÁFICAS}

Aburto R, I. (2004). Evaluación de 18 clones de yuca (Manihot esculenta). Presentación en el LI reunión del PCCMCA, Montelimar, Managua - Nicaragua. INTA, Pacífico Norte.

Cairo, P. (1995). La Fertilidad Física del suelo y la Agricultura Orgánica en el trópico. Universidad Nacional Agraria, Facultad de recursos Naturales y el Ambiente curso de post-grado, universidad central de las villas Cuba, 7-18 agosto 1995.

Chavarría, E. (2003). Evaluación agronómica de siete variedades de yuca (Manihot esculenta Crantz )en las condiciones del municipio de Nueva Guinea, Nicaragua $2002,93 p$.

CIAT (Centro Internacional de Agricultura Tropical). 1982. Yuca investigación, producción y utilización, Cali, Colombia. CIAT-PNUD; 660p.

CIAT (Centro Internacional de Agricultura Tropical). 1987. Informe anual, Cali, Colombia. CIAT P A-73.

Cock, j; Sandoval, D; Jori, P. (1978). Cassava o idiotype for maximum production.

Connor, DJ, H, Cock. (1981). The response of cassava to water shortage. II. Canopy dynamics. Field creeps Research. 4:285-296.

FAO (Organización de las Naciones Unidades para la Agricultura y la Alimentación) 2000.

Forsythe, W. (1980). Física de suelos, Manual de laboratorio. Editorial IICA. San José Costa Rica. 212 p.

INEC (Instituto Nicaragüense de Estadísticas y Censo). (2001). Reporte 2001. Managua, Nicaragua.

INETER (Instituto Nicaragüense de Estudios Territoriales) 2006. Reporte anual. Managua, Nicaragua.

Irikura, Y; Cock, JH; Kawano K. (1979). The physisological basis of genotype temperature interactions in cassava. Field Crops Research. 2: 227-239.

Montaldo, A. (1991). Cultivo de raíces y tubérculos Tropicales, San José Costa Rica. Segunda edición, publicado por Instituto Interamericano de Cooperación para la agricultura (IICA) 1991, 408 p.

NCR. 230:(1989). Norma de Costa Rica para la preparación y presentación de las Normas Nacionales.

Ospina, B; Ceballos, H. (2002). Sistemas modernos de producción, procesamiento, utilización y comercialización CIAT Colombia 586p.

Quintana, J. Blandón O, J, Flores, A; Mayorga, E. (1983). Manual de fertilidad para los suelos de Nicaragua. Editorial Primer Territorio Indígena Libre de América Ithaca, Nueva Your. Residencial Las Mercedes \# 19-A. Managua, Nicaragua, 60 Pág.

Sampat; Gavande (1991). Física de Suelos Principios y Aplicaciones. LIMUSA editorial S.A. de CV. México D.F. Primera Edición 1972, séptima reimpresión 1991, 351 p.

Singh, KD. (1970). Note on the effect of varying stages of harvest on tuber yield and starch content in different strains of cassava. Indian Journal of Agronomy (New Delhy 15(4) p (385-386).

Statistic Analisys System, SAS Institute V-9 Cary North Carolina, USA 2007. 